\section{Structure of the Early Universe Detected}

G.A. Tammann, Director of the Astronomical Institute, Basel, discusses the discovery by NASA's COBE spacecraft of fluctuations in the cosmic microwave background.

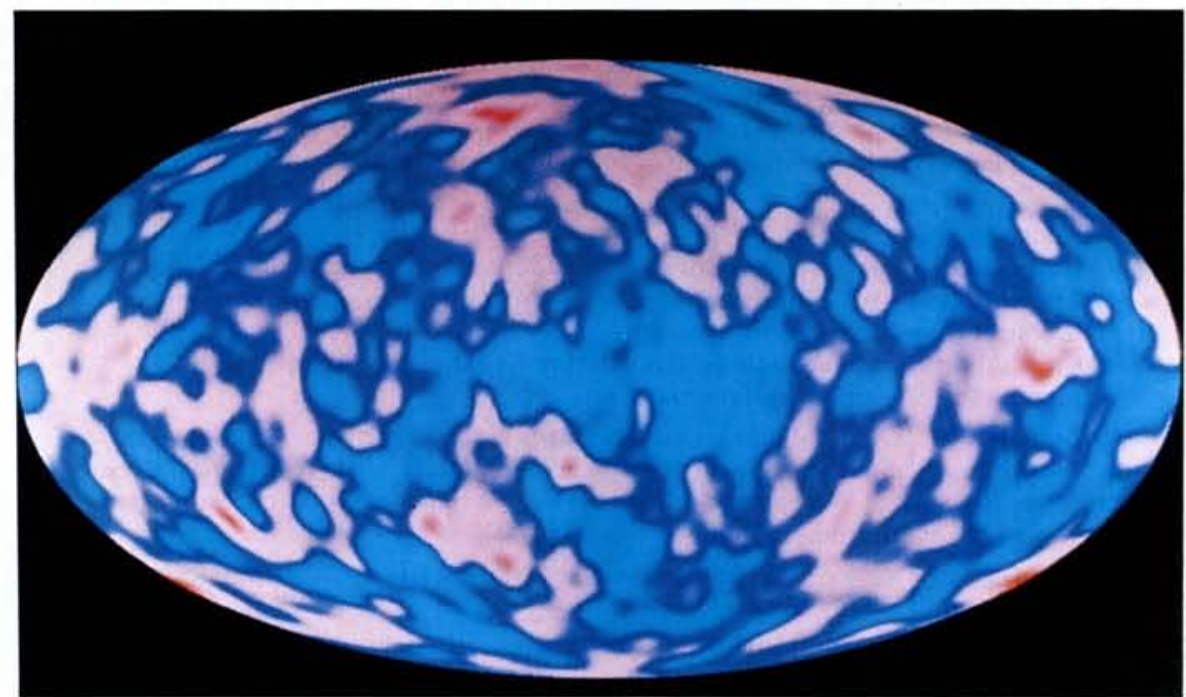

A full-sky COBE microwave map, in galactic coordinates (with our galaxy at the centre), after one year of data taking. Red (blue) indicates $0.01 \%$ warmer (colder) than the mean sky temperature of $2.73 \mathrm{~K}$ : most of the coloured patches represent instrumental noise. Computer analyses give intrinsic fluctuations of $0.0011 \%$. None of the map's features can be pointed to as representing these scale-invariant fluctuations, which are hidden in the noise with scales $\gtrsim 500 \mathrm{MPc}\left(4^{\circ}\right)$.

Big Bang models for the formation of the Universe remain stronger than ever because the glow left behind after the primordial explosion accounts for the cosmic microwave background $(\mathrm{CMB})$ radiation permeating the Universe. Solid evidence for this radiation was first reported by Penzias and Wilson, the Nobel Laureates, in 1965. The radiation is seen as coming from the surface of a spherical, thermally equilibrated, black body which existed, some 15000 million years ago, and which eventually became our world. Photons could not travel freely beyond this so-called diffusion sphere so it was opaque to electromagnetic radiation. Deeper and hence earlier structure therefore remains invisible.

By the mid-1970's, astrophysicists realised that the CMB should not be perfectly isotropic as there must be "seeds" to clump matter into clusters and individual galaxies. Indeed, such early seeds leading to gravitational potential fluctuations are predicted by some theories. For instance, the still speculative, inflation theory for the Universe predicts these seeds originated $10^{-35} \mathrm{~S}$ after the Big Bang.

Efforts to detect fluctuations in the $\mathrm{CMB}$ by measuring temperature differences $\Delta T$ between points in the sky subtending various angles using ground-based, rocketborne and balloon experiments were unsuccessful. Hence the considerable excitement accompanying the announcement on 22 April at the APS Spring Meeting in Washington that the Differential Microwave Radiometers (DMR) experiment on NASA's Cosmic Background Explorer (COBE) satellite (see box) had detected the long-sought large-scale fluctuations after a year's data taking. There is also much relief since what was expected for so long by so many had at last been found. One can now move forward. Indeed, the COBE team responsible for the discovery writes that "a new branch of astronomy has commenced" [E.L. Wright et al., submitted to Astrophys. J. Lett.; G.F. Smoot et al., submitted to Astrophys. J. Lett.].

\section{An Inflationary Universe}

COBE's latest large angular scale CMB anisotropy measurements reveal much more than the less precise preliminary data reported last year. Careful and detailed processing of the DMR data was needed to convert measured differences in the antenna temperatures to obtain the noisesubtracted $\mathrm{CMB}$ fluctuations. Correction factors include instrumental imbalance, emission from the Moon and from Jupiter (the main contribution from our Galaxy), the Doppler effect arising from the moving spacecraft, and the effects of the Earth's rotation and magnetic field.

Typical all-sky maps (see figure) of 6144 pixels were obtained by fitting data to spherical harmonic expansions corrected for the Doppler effect arising from the motion of the Earth relative to the CMB rest frame. This motion shows up as the well-known CMB "dipole" where the radiation intensity is slightly higher than average in a northerly direction (the red pole) and slightly weaker in the opposite direction (the blue pole). The COBE team determined more precisely the dipole intensity and direction and then assumed that the entire dipole results from our Local Group's ("peculiar") motion of $630 \mathrm{~km} / \mathrm{s}$ relative to the cosmic radiation field - a motion that is thought to be induced by density fluctuations on the 6500 $\mathrm{km} / \mathrm{s}$ scale. The dipole and second-order kinetic quadrupole terms, and the mean amplitude, were removed from the maps. A final step was to smooth the maps with a $7^{\circ} \mathrm{FWHM}$ Gaussian and convolute with the $7^{\circ} \mathrm{FWHM}$ antenna beam.

Intrinsic fluctuations of $30 \pm 5 \mu \mathrm{k}(\Delta T / T$ $\left.=1.1 \times 10^{-5}\right)$ in the very cold sky $(T=$ $2.73 \mathrm{~K}$ ) were observed for Galactic latitudes greater than $20^{\circ}$ well removed from regions where microwaves coming from the Milky Way cause problems. The small amplitude explains why earlier experiments did not detect the large-scale fluctuations. The rms quadrupole amplitude is $13 \pm 4 \mu \mathrm{K}$, some 100 times smaller than the dipole.

The COBE team believes the fluctuations discovered are in fact intrinsic to the $\mathrm{CMB}$, although one cannot completely rule out hitherto undiscovered Galactic and extragalactic emission. The temperature fluctuations and their angular spectrum are consistent with inflationary models of the Universe. They suggest structures of about $500 \mathrm{Mpc}$ in size, i.e., considerably larger than any galaxy clusters or superclusters detected so far.

Most of the evidence in favour of the inflationary model stems from the shape of the fluctuation power spectrum provided by the correlation function of fluctuations at the three wavelengths monitored. A powerlaw curve fits the primordial density fluctuation spectra. But the functions can also be fitted with other power spectra (e.g., Gaussian) of the small amplitude gravitational potential fluctuations so it remains an open question whether the smaller length scale portion of the spectrum is responsible for the development of the large-scale structure we see today.

\section{The COBE Spacecraft}

NASA's COBE spacecraft, launched in November 1989 into a polar orbit, scans the complete sky with a $7^{\circ}$ angular resolution every six months in a direction pointing away from the Earth and keeping the Sun at $100^{\circ}$ to shield terrestrial and solar radiation. The satellite's Differential Microwave Radiometers (DMR) experiment, one of three experiments, operates at three wavelengths each with two nearly independent channels. It measures differences in temperatures between two antenna $\left(7^{\circ}\right.$ beam) pointing into the sky at angles separated by $60^{\circ}$. By recording the amplitudes of spherical harmonics with I $<20$, DMR is sensitive to large-scale fluctuations in the cosmic microwave background radiation. System errors are typically $<8.5 \mu \mathrm{K}$ for smoothed rms sky fluctuations estimated to be $30 \pm 5 \mu \mathrm{K}$. 


\section{A New Astronomy}

The COBE results were compared with predictions of several simple models for primordial density fluctuation spectra. As the amount of gravity provided by the observed fluctuations is inadequate for galaxy and cluster formation, the normal assumption is that most of the material in the Universe is invisible, at least at present. For example, the "standard" cold (non-relativistic) dark matter model (CDM) only works if CDM is distributed in the same way as luminous matter (i.e., the bias factor $b_{\mathrm{s}}=1$ ). But if this is the case then one predicts peculiar motions which are very much larger than the observed small-scale velocity dispersions $\Delta v / v$ of less than 0.1. So CDM with $b_{\mathrm{s}}$ $=1$ is ruled out, illustrating that there are now some boundary conditions for eliminating several of today's dark matter dominated models for cosmological evolution. There are also indications that models based on large-scale textures in the Universe can also be rejected as they give much too large CMB fluctuations.

If the COBE results are correct, then several experiments (e.g., balloon measurements on $2^{\circ}$ scales by MIT and observations at the South Pole by a University of California, Santa Barbara, group) are close to detecting $\mathrm{CMB}$ fluctuations. A new branch of astronomy will then have been truly launched. The work at smaller angular scales will hopefully answer questions of galaxy formation where non-linear growth takes place as opposed to the initial perturbation spectrum addressed at large scales.

COBE will continue operating for a year or more to allow a two-fold reduction in noise that will reduce errors in the fluctuation measurements significantly. More accurate testing of evolutionary models will follow, notwithstanding that "damned small fluctuations mean things will be damned difficult". The next step would probably be an instrument with a much higher angular resolution than COBE. Perhaps this time there will be an European involvement.

\section{Science Policy and Management}

\section{PROCEEDINGS AVAILABLE}

The proceedings, edited by E.W.A. Lingeman, of the Europhysics Study Conference "Science Policy and Management" held at Schloss Dagstuhl, Germany, on 18-21 August 1991 can be ordered from the EPS Geneva Secretariat. The price is SFR 50. - plus postage (participants will each receive a free copy).

The proceedings represent a goldmine of information. They include invaluable and detailed presentations by representatves of nine major European research funding organisations together with analyses of general issues.

\section{Postdoctoral Positions for the STUDY OF WATER}

Three post-doctoral positions, each initially for one year with possible renewal for up to a further two years, will be available shortly in connection with an EEC funded collaboration involving United Kingdom, Italy, Spain, and Sweden. The planned research programme involves experimental neutron scattering, theoretical and computer simulation studies, and aims to make major advances in understanding the microscopic static and dynamic properties of water.

New neutron scattering data will be obtained by using the Isis spallation source at the Rutherford Appleton Laboratory. Computer simulations will be performed by using existing and newly developed potential models for water. Theoretical analysis will be used to assist in interpretation of both experimental and computer simulation data.

The positions will be based at:

1) Rutherford Appleton Laboratory, U.K. (experimental work)

2) The University of Trento and Istituto di Elettronica Quantistica, Firenze, Italy (computer simulation and theoretical work)

3) Royal Institute of Technology, Stockholm, Sweden (experimental work and data-handling).

Intended applicants should send a curriculum vitae to one of the addresses below as appropriate and provide evidence for their experience in a field related to the requirements of the project. Names and addresses of two referees who may be contacted should also be submitted.

Salary and allowances will be established according to age and experience in the field. Further details may obtained from:

Prof. Ulf Dahlborg, The Royal Institute of Technology,

Dept. of Nuclear and Reactor Physics, S-100 44 Stockholm (Sweden) fax: +46-8-7906396 — ulf@theophys.kth.se

Dr. Alan Soper, Isis Facility, Rutherford Appleton Laboratory, Chilton, Didcot, Oxon OX11 0QX (United Kingdom) fax: +44-235-445 720 — aks@de.rl.ac.uk

Prof. Renzo Vallauri (Coordinator), Università degli Studi, Dipartimento di Fisica, I-38050 Povo, Trento (Italy)

fax: +39-461-881696 - simulieq@ifiidg.cnr.it - itnvax::vallauri

Deadline for receipt of applications is 31st July 1992.

\section{THE UNIVERSITY OF GRONINGEN THE NETHERLANDS}

\section{announces an opening for a \\ Professor of Theoretical Condensed Matter Physics}

(vacancy number 920601)

The appointee is expected to play a leading role in setting up and supervising a research program in theoretical solid state physics and to provide theoretical support for research programs within the Materials Science Center (MSC). The MSC is an interdisciplinary research institute with a broad spectrum of basic material oriented research like surface physics, non-linear optics, exotic materials, high $\mathrm{T}_{\mathrm{c}}$ superconductors, thin films and multilayers, conducting polymers and magneto-optical materials.

The appointee is expected to play a leading role in a national effort to stimulate theoretical solid state physics. The appointee will also participate in teaching and policymaking activities.

Letters of application, including a curriculum vitae, a list of publications and the names and addresses of at least two referees should be sent within four weeks after publication of this advertisement to the Head of Personnel Department, University of Groningen, P.O. Box 72 , $9700 \mathrm{AB}$ Groningen, The Netherlands, quoting our reference number.

Further information can be obtained from the chairman of the appointment committee, Prof. Dr. G.A. Sawatzky phone, +31-50-634975 or from the chairman of the Physics Department, Prof. Dr. L. Niesen phone, +31-50-634735/634736.

Those whishing to recommend potential candidates are invited to send a letter to the Head of Personnel Department. 